\title{
Spectral Efficient Blind Channel Estimation Technique for MIMO-OFDM Communications
}

\author{
Renuka Bhandari, Sangeeta Jadhav \\ Department Of (E\&TC), Dr. D.Y. Patil Institute of Engineering \& Technology, Pimpri, Pune-411018 \\ Army Institute of Technology Pune-411015, India
}

\begin{abstract}
Article Info
ABSTRACT

Article history:

Received Jun 9, 2017

Revised Feb 20, 2018

Accepted Mar 11, 2018

\section{Keyword:}

Blind channel estimation

Error rates

Independent component

analysis

Interference

MIMO-OFDM

With emerge of increasing research in the domain of future wireless communications, massive MIMO (multiple inputs multiple outputs) attracted most of researchers interests. Massive MIMO is high-speed wireless communication standards. A channel estimation technology plays the essential role in the MIMO systems. Efficient channel estimation leads to spectral efficient wireless communications. The critics of Inter-Symbol Interference (ISI) are the challenging tasks while designing the channel estimation methods. To mitigate the challenges of ISI, we proposed the novel blind channel estimation method which based on Independent component analysis (ICA) in this paper. Proposed channel estimation it works for both blind interference cancellation and ISI cancellation. The proposed Hybrid ICA (HICA) method depends on pulse shape filtering and ambiguity removal to improve the spectral efficiency and reliability for MIMO communications. The Kurtosis operation is used to measure the complex data at first to estimate the common signals. Then we exploited the advantages of 3rd and 4th order Higher Order Statistics (HOS) to priorities the common signals during the channel estimation. In this paper, we present the detailed design and evaluation of HICA blind channel estimation method. We showed the simulation results of HICA against the state-of-art techniques for channel estimation using BER, MSE, and PAPR.
\end{abstract}

Copyright (C) 2018 Institute of Advanced Engineering and Science. All rights reserved.

\section{Corresponding Author:}

Renuka Bhandari,

Department Of (E\&TC), Dr. D.Y. Patil Institute of Engineering \& Technology,

Pimpri, Pune-411018, Army Institute of Technology Pune-411015, India.

Email: bhandarirenuka33@gmail.com

\section{INTRODUCTION}

Since from last decade, continues growth in the requirements for higher data rates on constrained resources and available bandwidth. This demand for higher data rate is resulting in the significant attention of researchers to initiate the working towards future wireless communications [1]. In future wireless communications, the resource utilization using MIMO (Multiple Input Multiple Output) is better than other methods [2] [3]. Hence massive MIMO is the crucial technology for future communication systems like 5G. The MIMO combined with transmission method like OFDM (orthogonal frequency division multiplexing) also the CDMA (code division multiple access). The critical part for MIMO systems is the design of efficient channel estimation to improve the overall communication performance and minimize the error rates. Therefore, several channel estimation methods designed in literature. The methods reported in [4-7] depend on ICA and wavelet-based channel estimation for MIMO wireless systems.

The channel estimation techniques of MIMO-OFDM divided into the three types such as semi-blind, blind channel, and training based evaluation methods [4]. In training based techniques, plan known training 
samples used to perform the extensive channel estimations. The least square (LS) and MMSE are the cases of training based channel assessments techniques. In blind channel estimation techniques, SOS (second-order stationary statistics) or HOS techniques used for transporting maximum spectral efficiency. In the semi-blind channel estimation, the main order statistics utilized. The semi-blind channel estimation technique consolidated attributes of training based and blind based channel estimation are interconnected with MIMOOFDM respectively. The semi-blind strategy sets aside longer time for the channel estimation and subsequently increases the overall communication costs. The blind channel estimation technique is superior to other two types of channel estimation methods. Thus in this paper we focused on designing the novel blind channel estimation method.

In OFDM systems, block-based symbol transmission method used; hence for 4G communications, the technique of block-based and iterative channel estimation introduced. For future wireless communication systems, same methods will be applicable. Therefore, it motivates to use blind channel estimation to obtain the initial symbol estimation and furthermore it is utilized the initial symbol evaluation to increases the more considerable dedication channel estimations. This channel estimation process repeated iteratively with soft information exchange to enhance the performance of channel estimation and data symbol estimations [8]. Further, consequently prompts the fast increment in low mobility based application and hence influence to design the blind channel estimation which requires a large number of samples based on quasi-static channel conditions.

The current investigations exhibited the SOS and HOS based blind channel estimation techniques. However, none of such process equipped for interference signals cancelation for MIMO-OFDM [11]. An interference signal generates the blind channel or scalable client in MIMO-OFDM wireless systems. The HOS relies upon ICA strategy for interference cancelation in MIMO systems, yet not expressly outlined and tended to for blind channel estimation. Moreover, ISI (inter-symbol interference) likewise have the critical effect on the performance of blind channel estimation and spectral efficiency. In this paper, we proposed distinctive spectral efficient channel estimation based on modified ICA approach called HICA. The proposed HICA method designed to mitigate the challenges of blind interference, ISI cancellations, and error minimizing efficiently using the pulse shape filtering, Kurtosis, HOS functions, and ambiguity removal. We present the extensive simulation analysis for proposed blind channel estimation method using BER, MSE, and PAPR performance metrics. The PAPR evaluation for MIMO systems recently introduced in [13]-[18], hence we conducted the PAPR performance investigation in this paper. In part II, the brief literature of past works exhibited, In part III, the proposed method for blind channel estimation discussed. In part IV, the simulation outcomes illustrated. The conclusion based on the results presented in section V.

\section{RELATED WORK}

The channel estimation is the crucial phase of wireless communications; designing channel estimation for MIMO gains the significant attention since from last decade. We discussed some of the recent methods presented for channel estimation as well as PAPR analysis.

The blind channel estimations by using the repeated index approach reported in [8]. They proposed subspace blind channel assessments design relies upon the rehashed list with the same yield from separates to ultimately strategies with a few quantities of symbols. Our work is not the same as this work as we in light of altered ICA way to deal with blind interference cancellations and error minimization.

In [9], another blind channel estimation technique in the MIMO-OFDM system with the equilateral spacetime piece code investigated. This approach composed the new weighted covariance matrix of data recognize in the direction to obtain the errors in the code. All the non-scalar ambiguities are resolved using method reported in [9], but failed to address the challenges of common signal estimation and prioritizing the estimated common signals.

Further, the SOS based blind channel estimation method reported in [10] based on the novel blind recursive approach in MIMO-OFDM systems. The subspace based tracking designed for the fast varying wireless channels. To get SOSs update fast, data used from time and frequency domain due to frequency correlation of this wireless channels.

In [11], the blind channel estimation technique using the SOS and subspace models for the MIMOOFDM system reported. Authors efficiently utilized the null space produced by OSTBC. The method designed with the goal of minimum received blocks. The experimental results demonstrated on the single input and single output only. Our approach depends on multiple inputs and multiple output antennas for the channel estimation.

Another recent approach for channel estimation reported in [12]. The blind channel estimation techniques utilized for the LTE (Long-Term Evaluation) networks. The channel estimation performed using the wavelet transform with ICA. The wavelet transforms used preceding ICA channel estimation to diminish 
the errors in approaching signals. The denoising approach was intended to deal with the blind interference cancelation. It is exploiting the ICA. The ISI cancelation techniques are not utilized in this technique.

In this paper, we proposed the novel channel estimation strategy which differs from previous works of channel estimation discussed above. The proposed blind channel estimation method based on ICA aims to ISI and blind interference cancelations. At first, to mitigate the noise interference and ISI effect, we designed the pulse shape filtering to apply on modulated signals. At channel estimation phase, the source of signals estimated using the kurtosis values, then common signals are detected and then prioritize common estimation signals using 3rd and 4th order HOS. The lightweight method used for the ambiguity removal.

Additionally, we presented the PAPR evaluation of proposed blind channel estimation technique for MIMO-OFDM systems in this paper. The PAPR analysis of space-time block-coded (STBC) MIMO-OFDM method for the 4G wireless networks evaluated in [13]. The numbers of techniques introduced to decreases PAPR in the (STBC) MIMO-OFDM system such as SLM, clipping and filtering and partial transmit sequence. Their simulation outcomes claim that clipping and filtering delivered effective PAPR reduction than the others methods and only SLM technique conserves the PAPR reduction in reception part of the signal. We studied some recent works reported for PAPR performance evaluation in [14]-[18].

\section{SYSTEM MODEL}

The system model for proposed HICA channel estimation is showing in Figure 1 for MIMO-OFDM systems.

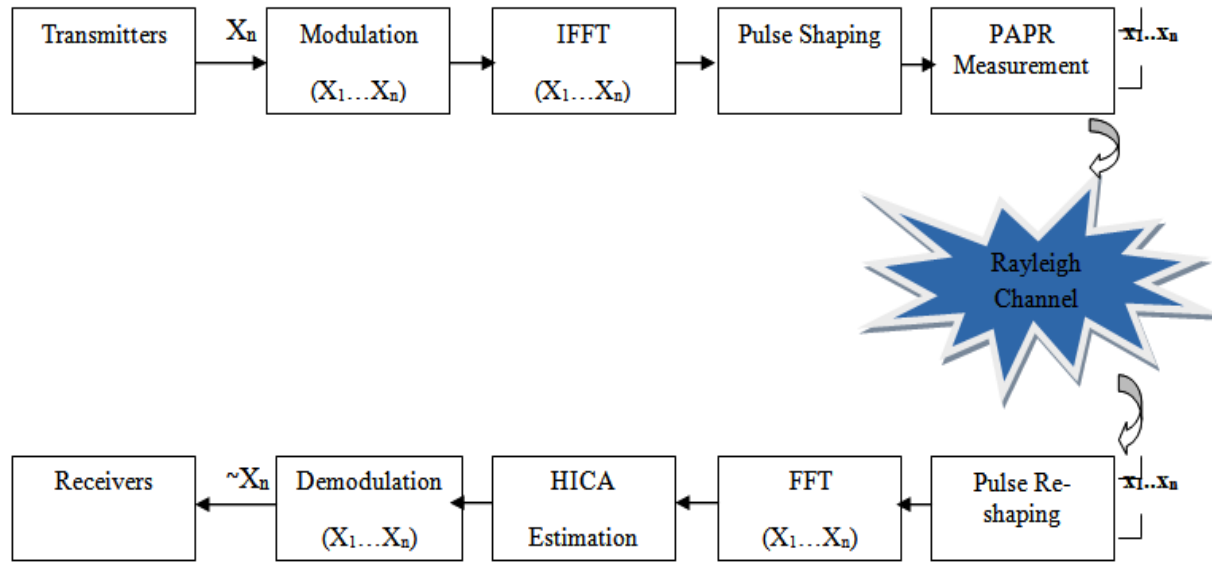

Figure 1. Proposed MIMO-OFDM System Model

In proposed system model, at transmitter data $\mathrm{X}$ from users is randomly generated and then forwarded to modulation process on each symbol. IFFT and CP operations performed at each transmitter. To mitigate the effect of ISI cancellation, we applied the pulse shape filtering on IFFT data. Once symbols are ready to transmit over the Rayleigh channel through transmitter's antennas towards receiver's antennas, the measurement of PAPR performs on each symbol. At the receiver side, after the pulse reshaping, CP removal and FFT operations, proposed HICA method for efficient channel estimation and signal detection performed. Figure 2 showing the complete functionality of HICA block. After estimation, the reverse operations applied at the receiver side to get the original data at the receiver side. The modulation and demodulation are performed by using QPSK technique. Let's consider S is the sample of signal, $\mathrm{M}$ is a transmitted signal which include the many numbers of samples. An is an attenuation for $n$th channel. The Factor of $A n$ is complex number. The authors investigates the pulse shaping for the deleting the ISI impacts to reduces the error rates and optimize the spectral efficiency. The HICA method for blind channel estimation consists of steps like source estimation, common signal estimation; prioritize the signals, ambiguity removal, and final signal detection at the receiver side. The functionality of complete architecture presented in the form of algorithms below. 


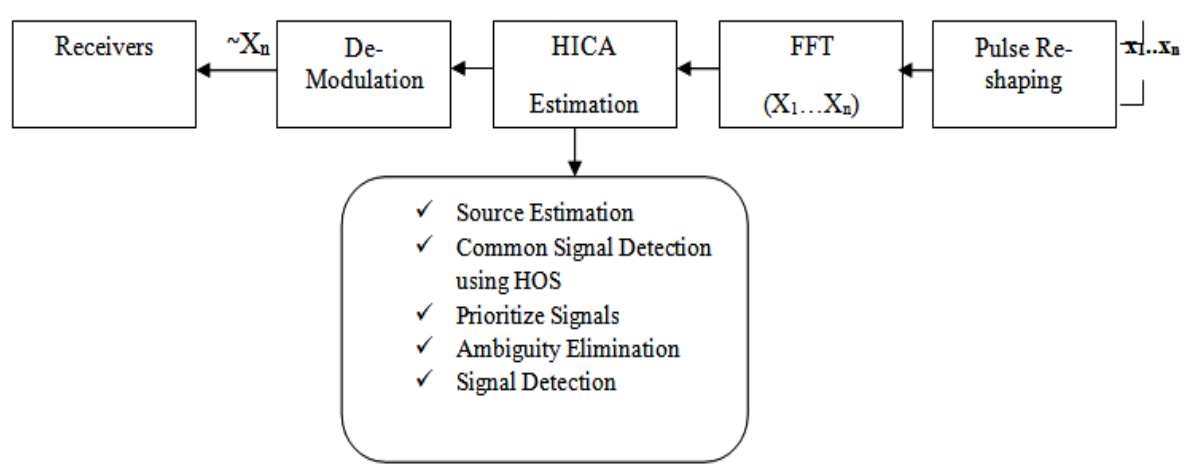

Figure 2. HICA channel estimation phases

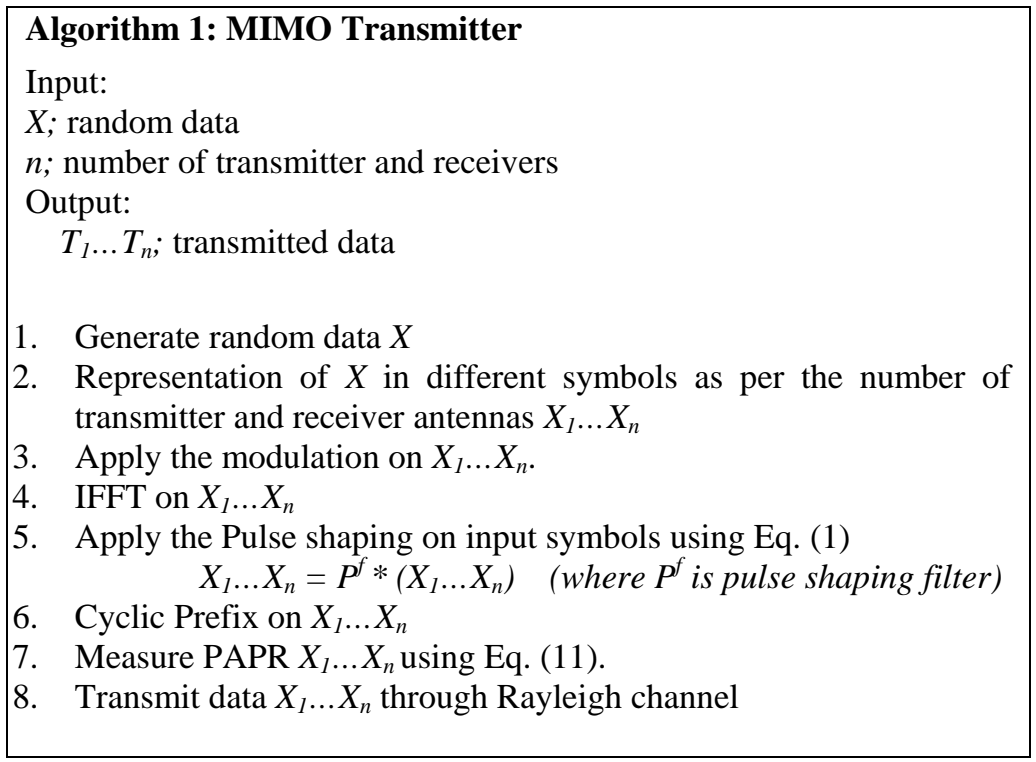

Algorithm 2 is showing the process at receiver side.

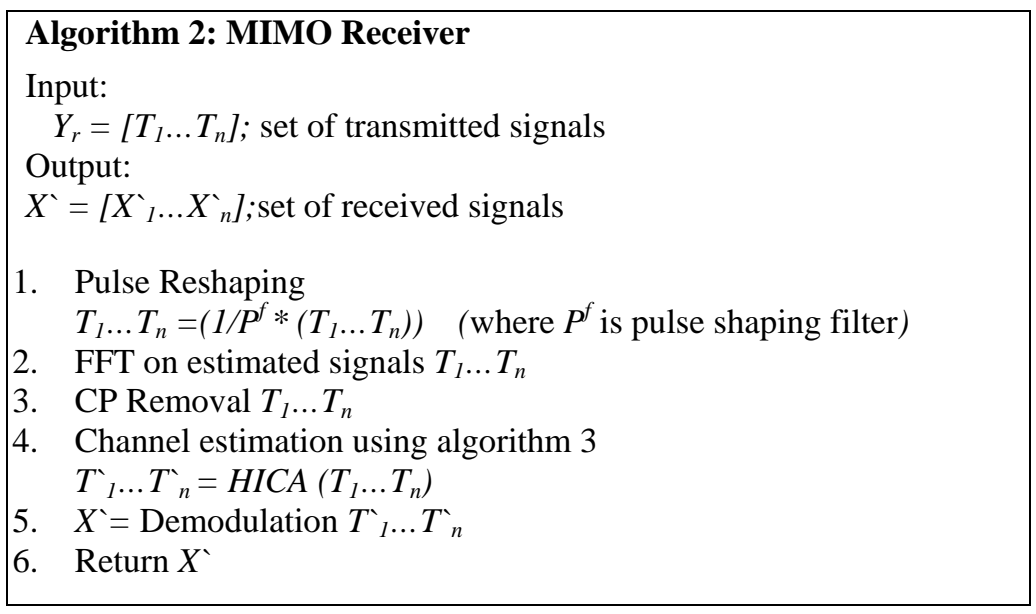

The algorithm 3 shows the process for proposed channel estimation method called HICA. The proposed channel estimation approach is the iterative process to optimize the channel estimation performance and minimize the errors of estimation. 


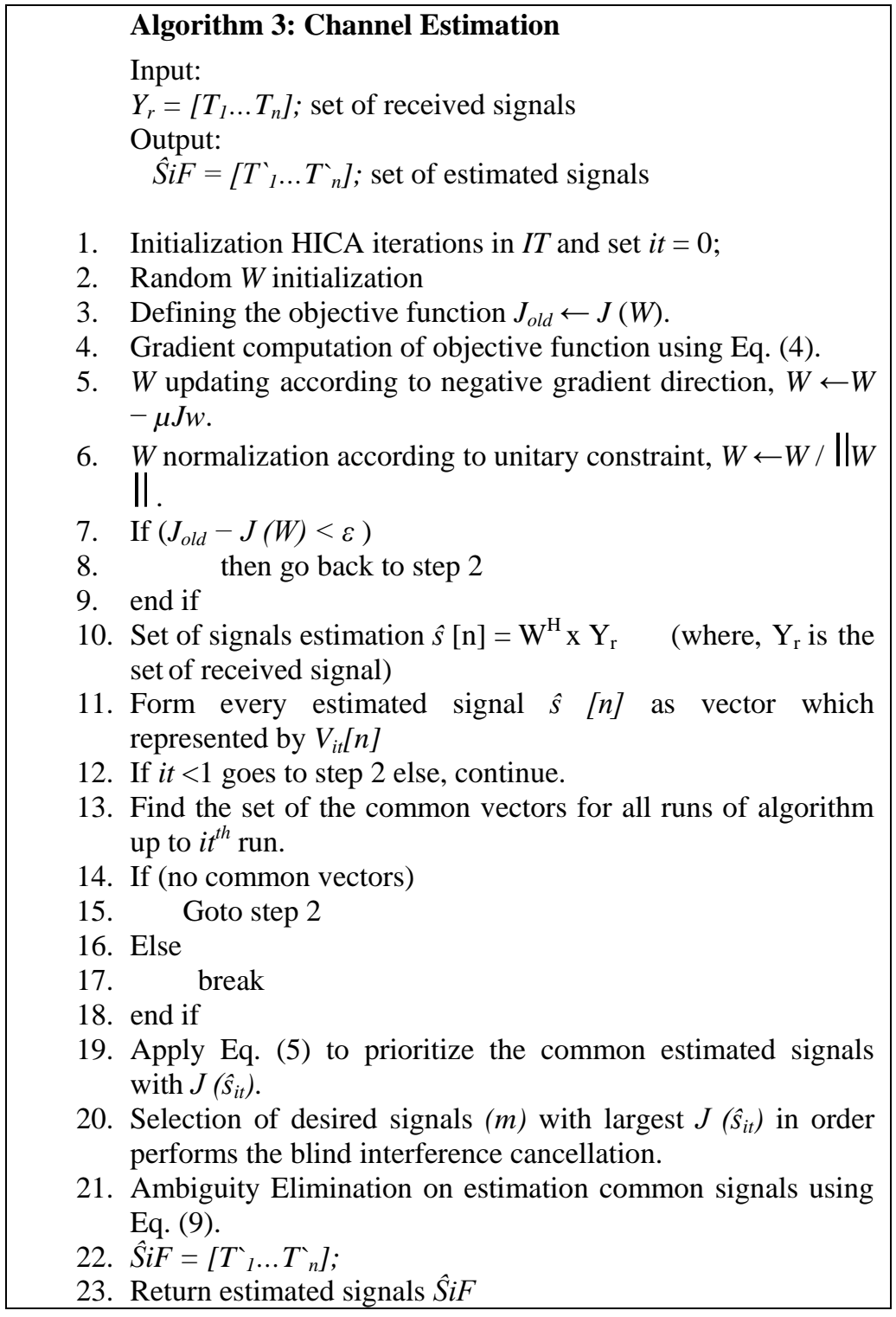

\section{Algorithm 3 HICA}

In the proposed method, we first enable the pulse shaping techniques in each user symbol in the order to minimize the internal symbol interference at the transmitter side. Pulse shape is simple ISI cancellationtechniques. We utilized the square root increased cosine filter in sampling region to perceive the pulse shaping filter. Table 1 shows the symbols used by proposed channel estimation method.

Table 1. List of Symbols

\begin{tabular}{cl}
\hline Symbol & \multicolumn{1}{c}{ Meaning } \\
\hline$g_{t}(t)$ & Transmitter side pulse shape filter for each symbol \\
$\mathrm{g}_{\mathrm{r}}(\mathrm{t})$ & Receiver side pulse shape filter for each symbol \\
$T$ & Number of transmitters \\
$R$ & Number of Receivers \\
$L$ & Number of taps \\
$i_{R}$ & $\mathrm{i}^{\text {th }}$ receiver \\
$i_{T}$ & $\mathrm{i}^{\text {th }}$ transmitter \\
$W$ & Separation Matrix \\
$v$ & non-gaussianity for random variable \\
$K$ & Kurtosis \\
\hline
\end{tabular}


The composite channel is represented as $T * R$ with matrix $H(t)$. The $\left(i_{R}, i_{T}\right)$ channel using pulse phase filtering is represented by:

$$
h_{i R^{\prime}} i_{T}(\mathrm{t})=h_{i R^{\prime}} i_{T^{\prime} c}(t) * g_{t}(t) * g_{r}(t)
$$

Where $\mathrm{h}_{\mathrm{i} \mathrm{R}^{\prime}} \mathrm{i}_{\mathrm{T}^{\prime} \mathrm{c}}(\mathrm{t})$ is the (iR, iT) element of $H(t)$. Here the channel can be represented as the $L$ tap FIR filters array for blind channel estimation [19]. After the designing of pulse shaping filter which we applied to time domain (i.e., after IFFT operation) and perform the subsequent tasks of transmitter side. The data is transmitted from the transmitter's antennas towards the receiving antennas via Rayleigh channel.

At receiver end, the channel estimation is performed after the pulse re-shaping, FFT and cyclic prefix removal operations (Algorithm 2). For the channel estimation, we proposed the algorithm 3 called HICA.

As observed in algorithm 3, initially signals sources from the receiver analysis mixture are determine by using the partition of matrix $W$. The proposed channel estimation algorithm is based on the iterative process for excellent channel estimation, thus at each iteration, the order of initial signal estimation is varying due to the random initialization of separation matrix. But, if the important information discovered in estimated signals, then it will be utilized for the iteration. The identified important information called as common estimated signals. The HOSs 3rd and 4th order derivatives applied to the common estimated signals to prioritize them. The outcome of HOS is the selected desired signals $(m)$ by canceling the interference components

from the signals.

The objective function based on $W$ introduced based on the principle of non-gaussianity maximizing observation signals. The outcome of non-gaussianity for random data $(v)$ containing the complex data which is measured by the operator Kurtosis $K[s]$ :

$$
K[s]=E\left[|v|^{4}\right]-2\left(E\left[|v|^{2}\right]\right)^{2}-E[v \cdot v] E\left[v^{*} v\right]
$$

Where $(.)^{*}$ is represents the complex conjugate. Before prioritising the common estimation signals, the Kurtosis function is applied to discover the common estimation signals. The signal estimation using $W$ based on the minimization of objective function $J(W)$ within the unitary constraint

The estimation of $W$ is performed by the minimization of $J(W)$ objective function within the $W W^{H}$ $=I_{R}$ unitary constraint. The unitary constraint used to alleviate the negative outcomes of kurtosis value for various modulation methods. Thus, the objective function using the estimation signals $\hat{s}[n]$ kurtosis values represented by:

$$
W=\left\{\min _{W} J(W)=\sum_{j=1}^{M} K[\hat{s}[n]]\right.
$$

The minimization of objective function $J$ (W) satisfied using the gradient computation represented by:

$$
\left.\mathrm{JW}=\frac{\partial \mathrm{J}(\mathrm{W})}{\partial \mathrm{W}}=\mathrm{K}\left(\mathrm{W}^{\mathrm{H}} \hat{\mathrm{S}}[\mathrm{n}]\right)\left[\mathrm{E}\{\hat{\mathrm{s}}[\mathrm{n}])^{3}\right\}\right]
$$

Where $(w)$ is one of the vectors from $W$ separation matrix. According to the constraint for the objective function $(\mathrm{JW})$, the object function gradient complemented projecting $W$ over the interval after each step performed by dividing the $W$ by its norm.

The iterative execution using different random initialization matrix $W$ performed to evaluates the general signals at the receiver side. The two estimated signals of various computations are examine as different if the spectral angle mapper (SAM) of their vectors $v_{i}[n]$ and $v_{j}[n]$ is beyond the threshold value $\varepsilon$. The general signal estimation results used to prioritize the signals based on the $3^{\text {rd }}$ (Eq. 5) and $4^{\text {th }}$ (Equation 5) higher order statics.

$$
J\left(\hat{\mathrm{s}}_{q}\right)=\left(\frac{1}{12}\right)\left[Q_{q}^{3}\right]^{2}+\left(\frac{1}{48}\right)\left[Q_{q}^{4}-3\right]^{2}
$$

Where, $Q_{q}^{3}=E\left\{\hat{\mathrm{s}}_{q}^{3}\right\}=\left(\frac{1}{T}\right) \sum_{n=1}^{T}\left(\hat{\mathrm{s}}_{q}[n]\right)^{3}$ is the $3^{\text {rd }}$ order of statistics and

$Q_{q}^{4}=E\left\{\hat{\mathrm{s}}_{q}^{4}\right\}=\left(\frac{1}{T}\right) \sum_{n=1}^{T}\left(\hat{\mathrm{s}}_{q}[n]\right)^{4}$ is the $4^{\text {th }}$ order of statistics of estimated signals, and $q$ is the execution index. 
Once the signal estimated common signals prioritise, the next step of ambiguity removal applied to suppress the unwanted components from the estimated signals. Scientifically the estimated $\hat{s}_{i}[n]$ is not matching to the original transmitted $s[n]$ due to presence of ambiguity matrix $A$ in $\hat{s}_{i}[n]$. This can be represented as:

$$
\check{s}_{i}[n]=A \mathrm{X} \hat{s}_{i}[n]
$$

Two indeterminacies forming the $A$ as:

$A=P \times D$

Where $P$ is the permutation ambiguity matrix and $(D)$ is phase rotation ambiguity matrix.

The removal of such ambiguities performs to reduce the error rates in proposed channel estimation techniques. The ambiguities suppressed by multiplying the estimated signals and remove from the original signals in first step represented by $L_{F}$ :

$$
L_{F}=\arg \min _{L \mathcal{E} G}\|s[n]-\hat{s}[n] x \mathrm{~A}\|^{2}
$$

After the $L_{F}$ computation, the multiplying it with the estimated signals as:

$$
\hat{s}_{i F}[n]=L_{F} x \hat{s}[n]
$$

For the performance evaluation along with BER and MSE, we computed the PAPR for MIMO systems. The computation of PAPR is done as per given below:

The PAPR of the signal, $\mathrm{x}(\mathrm{t})$, is then provided the peak prompt power to the average power, following are shown the formula,

$$
\mathrm{PAPR}=\log 10 * \frac{\max 0 \leq t \leq T|x(t)|^{2}}{E\left[\left|x(t)^{2}\right|\right]}
$$

Where E [.] is the predicated operator. From the central limit formulas, for most values of N, the $\mathrm{X}$ (t) is a real and imaginary values. The PAPR computation showing in Eq. (10) is applicable for Single Input Single Output (SISO) systems, therefore in MIMO systems; same formula is utilized to compute the PAPR for each transmitter given by:

$$
P A P R_{M I M O-O F D M}=\max _{i \leq i \leq n_{t}} P A P R_{i}
$$

Where $P A P R_{i}$ denotes the PAPR of transmit $i^{\text {th }}$ antenna.

\section{SIMULATION RESULTS AND DISCUSSION}

The performance analysis of the innovated channel estimation technique for MIMO systems has been shown in this section against the state-of-art techniques. The simulation performed for this study under different network conditions such as varying number of sender antennas and accepted antennas. Table 2 shows the simulation parameters used to evaluate the proposed method. For comparative study, we used the conventional ICA technique [20] and recent wavelet denoising of ICA (WD-ICA) [12] method.

Table 2. MIMO-OFDM Simulation Parameters

\begin{tabular}{ll}
\hline \multicolumn{1}{c}{ FFT Size } & \multicolumn{1}{c}{$64,128,256$} \\
\hline Transmit Antennas & 2,4 \\
Receive Antennas & 2,4 \\
Block size & 8 \\
Sub band size & 20 \\
SNR level & $0: 5: 30$ \\
Number of iterations & 10 \\
Blind Estimation Method & ICA [20], WD-ICA [12] and Proposed HICA \\
Modulation Technique & QPSK \\
Number of subcarriers & 256 \\
Filter type & Pulse shaping filter \\
Oversampling factor & 4 \\
Channel Type & Rayleigh \\
\hline
\end{tabular}


To check the reliability of proposed channel estimation method, we performed the simulations based on (1) changing the number of transmitter and receiver antennas such as $2 \times 2$ and $4 \times 4$ and (2) varying FFT sizes such as 64,128 , and 256. The performance measured in three parameters such as BER, MSE, and PAPR to check the trade-off between spectral and PAPR efficiency for each investigated method.

\subsection{2x2 Transmit/Receive Antennas}

This section presents the simulation results for 2x2 (2-transmit and 2-receiving antennas) for MSE, BER and PAPR performance metrics for each varying FFT size.

The performance of MSE and BER is significantly improved by proposed HICA channel estimation method due to iterative error minimization approach using the Kurtosis and HOS functions for the common channel estimations. However, the performance PAPR is not promising for all the methods. In an average of 64 FFT size, WDICA method showed minimum PAPR as compared to ICA and proposed HICA method.

The Figures 6 to 8 shows the results using the FFT size 128. The PAPR ratio increased as the FFT size increases. The Figures 9 to 11 shows the BER, MSE and PAPR results using the FFT size 256. The proposed channel estimation method was demonstrating the superior performance against the state-of-art ICA based methods for the channel estimation using varying FFT size. However, the PAPR results show the proposed channel estimation method more PAPR rate as compared to conventional ICA and WD-ICA methods. Figure 11 shows the FFT size having the impact of PAPR performance, as the FFT size increases the PAPR is also growing. The result of BER and MER proves the proposed channel estimation techniques are the dependable techniques MIMO- OFDM systems. The impacts of FFT size on BER and MSE is showing in Tables 1 and 2 respectively.

Increasing FFT size leads to minimizing the BER and MSE values for all the methods as the number of subcarriers increases to transmit each symbol. However, the PAPR performance has the reverse impact as shown in table 3. Increased FFT size is showing the worst PAPR performance for all the methods. For each FFT size, the average PAPR result of WD_ICA having minimum PAPR rate. In this sub-section, we evaluated the impact of varying FFT size on BER, MSE, and PAPR performances using 2x2 transmit/receive antennas.

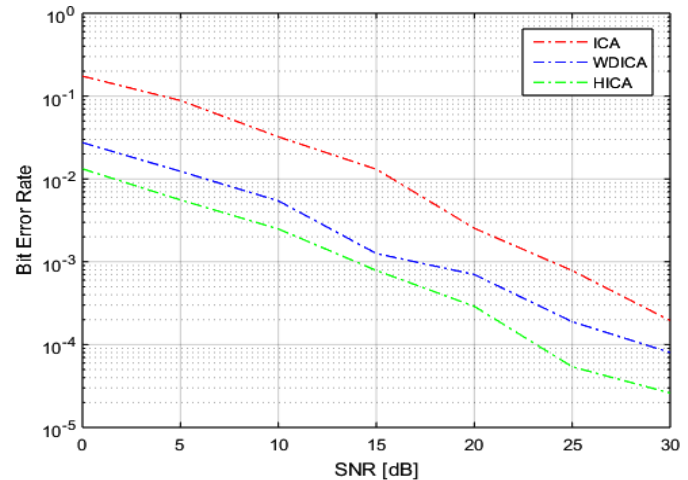

Figure 3. 2x2 BER analysis using FFT size 64

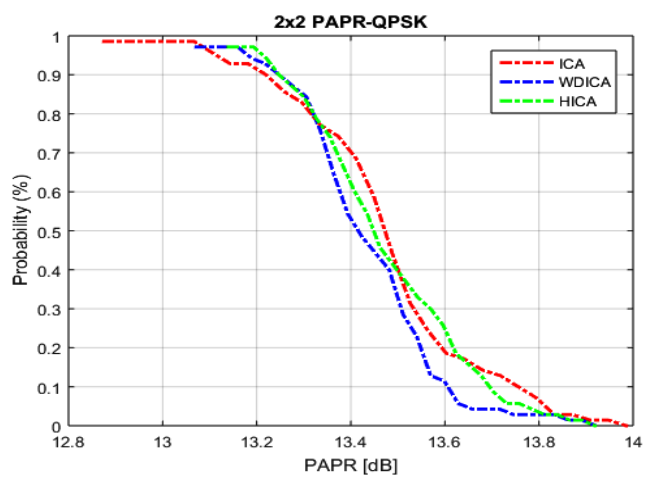

Figure 5. 2x2 PAPR analysis using FFT size 64

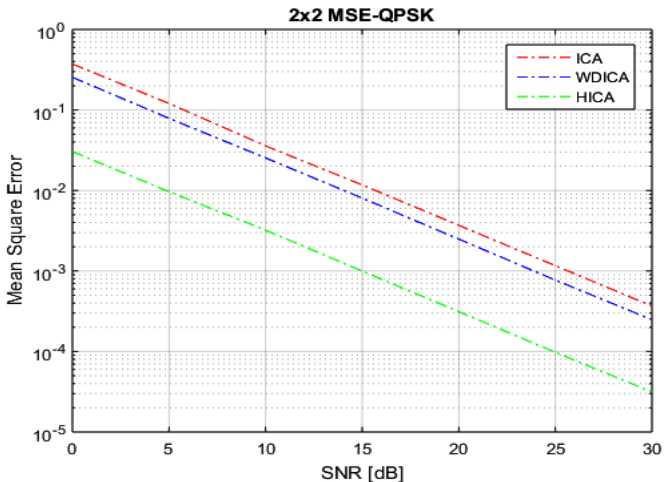

Figure 4. 2x2 MSE analysis using FFT size 64

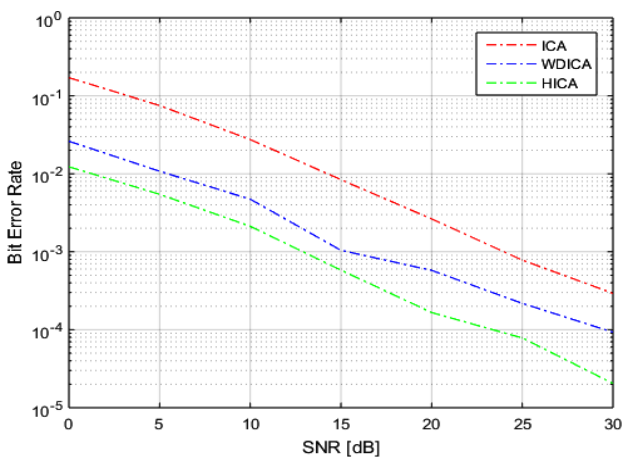

Figure 6. 2x2 BER analysis using FFT size 128 


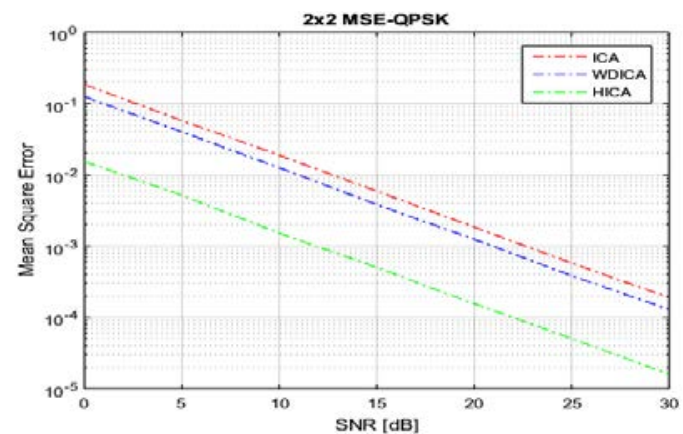

Figure 7. 2x2 MSE analysis using FFT size 128

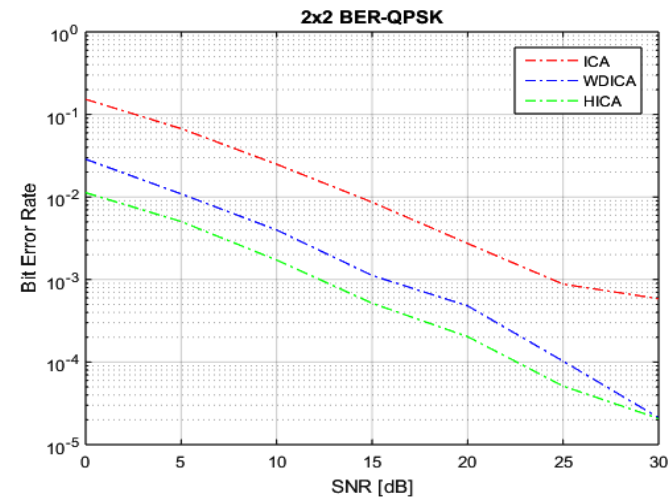

Figure 9. 2x2 BER analysis using FFT size 256

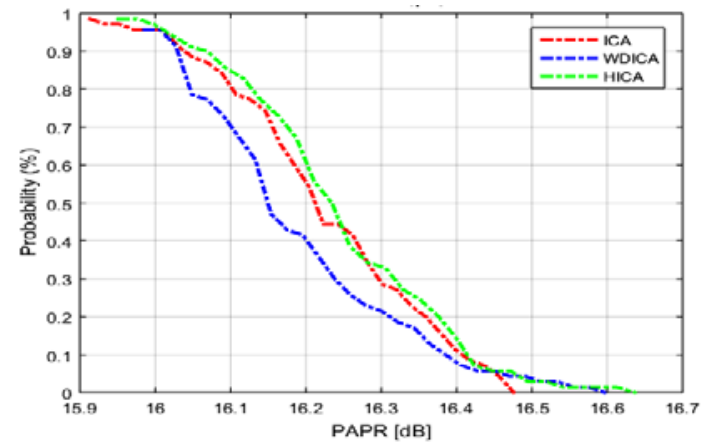

Figure 8. 2x2 PAPR analysis using FFT size 128

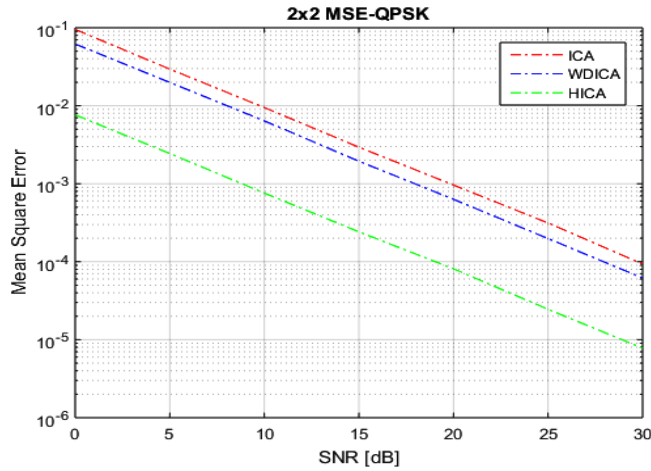

Figure 10. 2x2 MSE analysis using FFT size 256

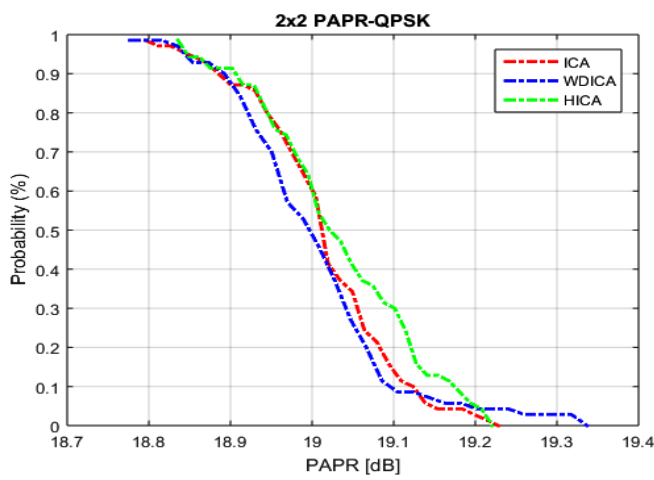

Figure 11. 2x2 PAPR analysis using FFT size 256

Table 3. Average BER Performance Analysis (2x2)

\begin{tabular}{llll}
\hline FFT Size & ICA & WDICA & HICA \\
\hline 64 & 0.0445 & 0.0068 & 0.0032 \\
128 & 0.0408 & 0.0062 & 0.003 \\
256 & 0.0368 & 0.0065 & 0.0027 \\
\hline
\end{tabular}

Table 4. Average MSE Performance Analysis (2x2)

\begin{tabular}{llll}
\hline FFT Size & ICA & WDICA & HICA \\
\hline 64 & 0.0779 & 0.0529 & 0.0064 \\
128 & 0.0385 & 0.0261 & 0.0032 \\
256 & 0.0198 & 0.013 & 0.0016 \\
\hline
\end{tabular}

Table 5. Average PAPR Performance Analysis (2x2)

\begin{tabular}{llll}
\hline FFT Size & ICA & WDICA & HICA \\
\hline 64 & 13.4883 & 13.4443 & 13.4823 \\
128 & 16.2325 & 16.1961 & 16.2552 \\
256 & 19.0180 & 19.010 & 19.0375 \\
\hline
\end{tabular}




\section{2. $4 \times 4$ Transmit/Receive Antennas}

After the investigation of FFT size on channel estimation methods, to claim the reliability and scalability of proposed channel estimation, we used 4x4 transmit/receive antennas for MIMO-OFDM communications. This section presents the simulation results for 4x4 (4-transmit and 4-receiving antennas) results for MSE, BER and PAPR performance metrics for each varying FFT size.

The varying transmitting and receiving antennas not having the significant impact on HICA performance. However, the HICA performance is significantly improved as compared to state-of-art methods using 64 FFT size (Figure 12 and Figure 13), using 128 FFT size (Figure 15 and Figure 16) and using 256 FFT size (Figure 18 and Figure 19). The channel estimation method should be lightweight and spectral efficiency. The results of MSE and BER show that HICA the spectral efficient way for MIMO-OFDM systems. The PAPR ratio is increased as the FFT size increases using $4 x 4$ antennas scenario as well.

The effect of increasing the antennas on MSE, BER and PAPR performances are review in Tables 6, 7 and 8 accordingly.

The increasing antennas from $2 \times 2$ to $4 \times 4$ does not show the significant impact of performances, except little increase in MSE and BER rates as the compared 2x2 scenario. The purpose of evaluating the channel estimation methods using different antenna sizes is to check the reliability of the proposed method. The results show that for all the cases of varying FFT size and antenna scenarios, suggested HICA method delivered efficient channel estimation performance as compared to state-of-art methods. There is little decrease in PAPR rate using $4 \times 4$ situations as compared to $2 \times 2$ scenarios. From all the results, the performance of MSE and BER are the contrast to PAPR performance for all the channel estimation methods. Achieving the trade-off between PAPR and error rates is one of the leading research problems for MIMOOFDM systems. Finally, Table 9 shows the average processing time performance for each channel estimation method. The results of processing time demonstrate the proposed method has very less computation overhead as differentiates to both state-of-art channel estimation techniques. The simulation conducted on the I5 processor with 4GB RAM.

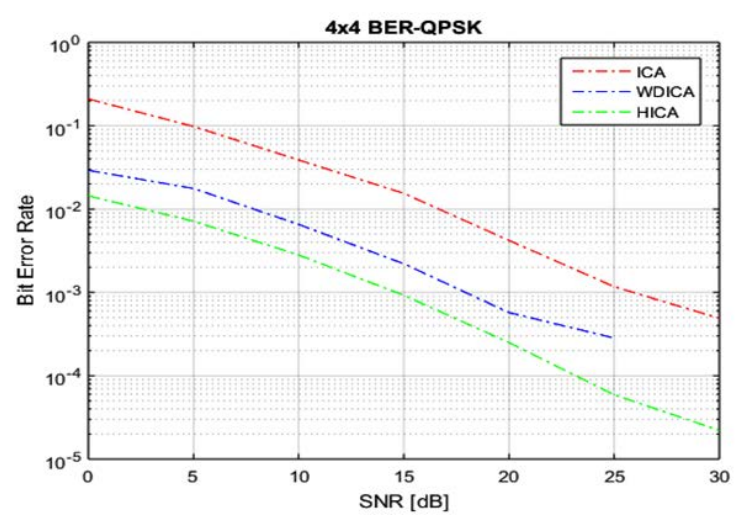

Figure 12. 4x4 BER analysis using FFT size 64

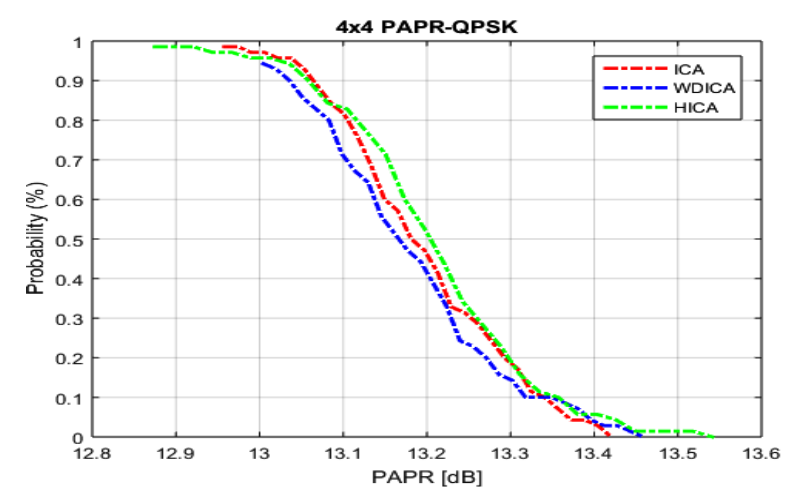

Figure 14. 4x4 PAPR analysis using FFT size 64

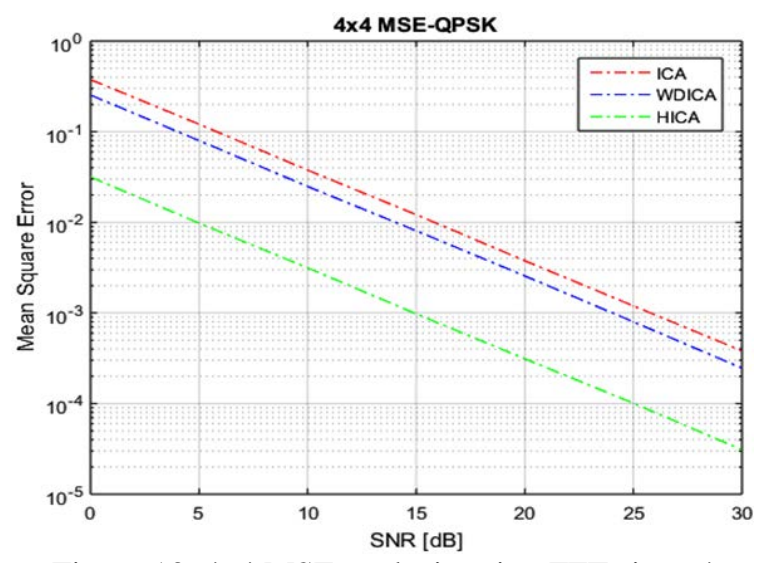

Figure 13. 4x4 MSE analysis using FFT size 64

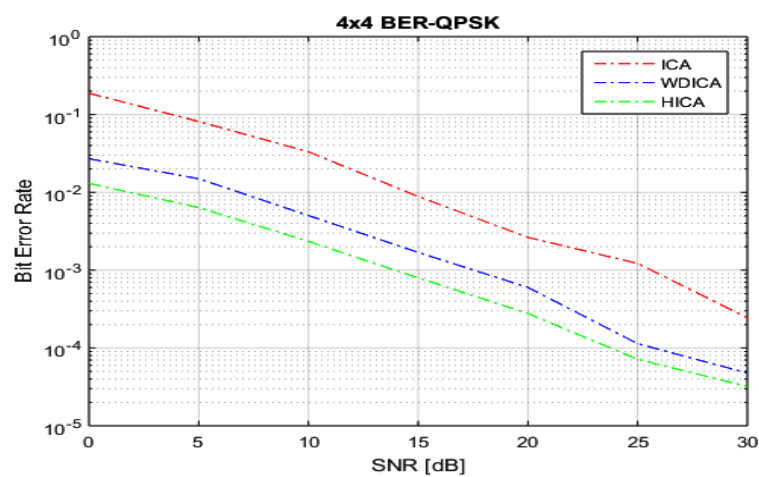

Figure 15. 4x4 BER analysis using FFT size 128 


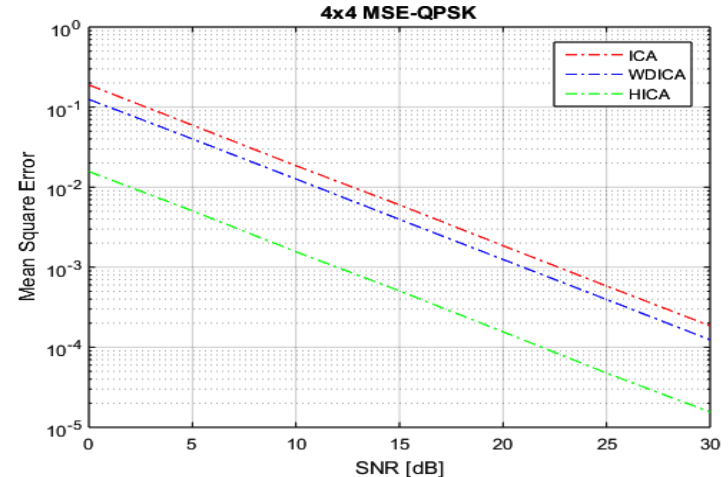

Figure 16. 4x4 MSE analysis using FFT size 128

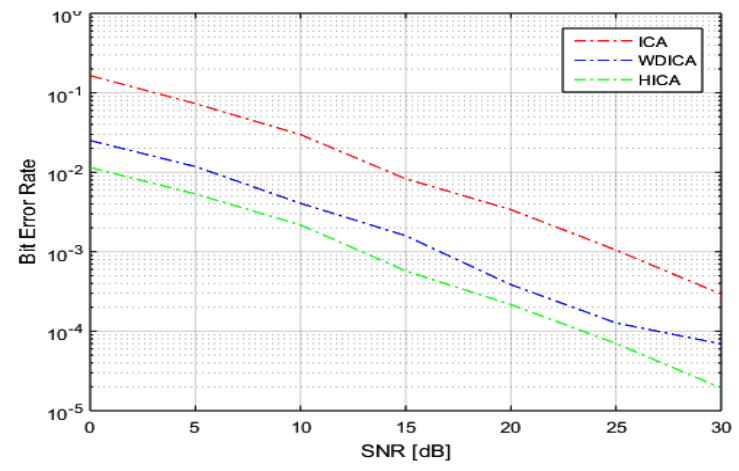

Figure 18. 4x4 BER analysis using FFT size 256

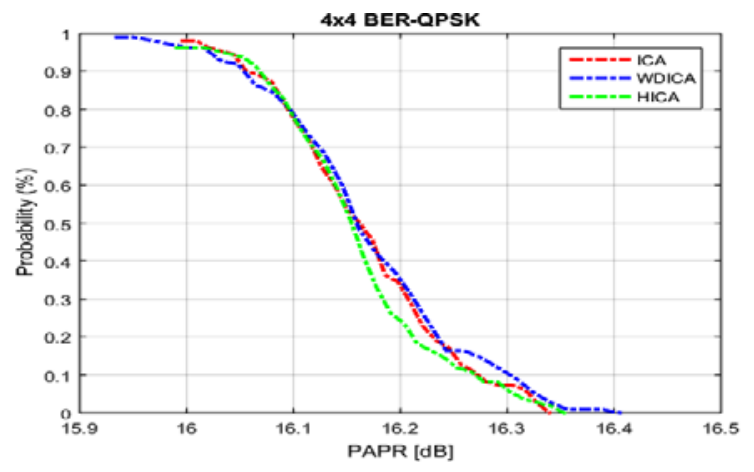

Figure 17. 4x4 PAPR analysis using FFT size 128

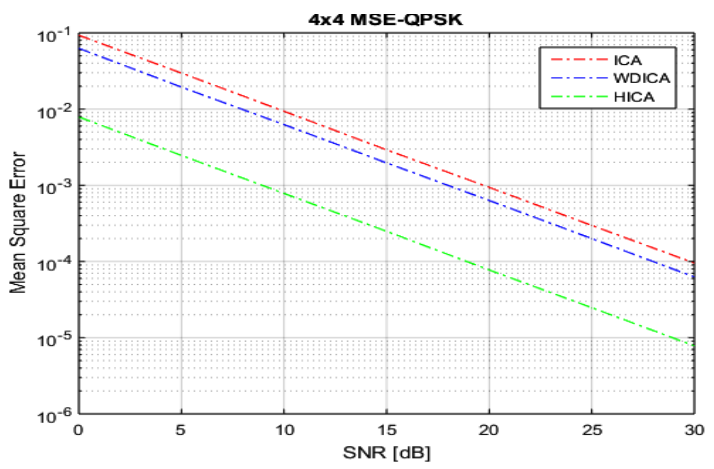

Figure 19. 4x4 MSE analysis using FFT size 256

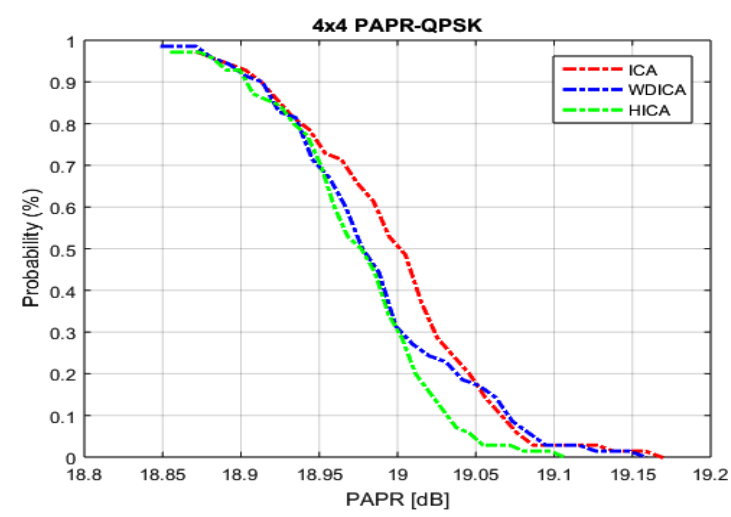

Figure 20. 4x4 PAPR analysis using FFT size 256

Table 6. Average BER performance analysis (4x4)

\begin{tabular}{llll}
\hline FFT Size & ICA & WDICA & HICA \\
\hline 64 & 0.0525 & 0.0081 & 0.0037 \\
128 & 0.045 & 0.0071 & 0.0033 \\
256 & 0.04 & 0.0062 & 0.0028 \\
\hline
\end{tabular}

Table 8. Average PAPR performance analysis $(4 \mathrm{x} 4)$

\begin{tabular}{llll}
\hline FFT Size & ICA & WDICA & HICA \\
\hline 64 & 13.2 & 13.18 & 13.21 \\
128 & 16.17 & 16.16 & 16.17 \\
256 & 18.99 & 18.98 & 18.97 \\
\hline
\end{tabular}

Table 7. Average MSE performance analysis $(4 \times 4)$

\begin{tabular}{llll}
\hline FFT Size & ICA & WDICA & HICA \\
\hline 64 & 0.0788 & 0.0528 & 0.0066 \\
128 & 0.0394 & 0.0261 & 0.0033 \\
256 & 0.0195 & 0.013 & 0.0016 \\
\hline
\end{tabular}

Table 9. Average processing time performance

\begin{tabular}{llll}
\hline Method & ICA & WD-ICA & HICA \\
\hline Time (Seconds) & 49.55 & 47.2 & 36.23 \\
\hline
\end{tabular}




\section{CONCLUSION AND FUTURE WORK}

In this research paper, we investigate the novel hybrid blind channel estimation technique based on modified ICA technique using pulse shape filtering approach. The HICA effectively utilized the Kurtosis and HOS features to estimate and prioritize the common signals respectively. The idea of ambiguity removal helps to reduce the error rates further. The proposed HICA method designed and evaluated with conventional ICA and recent WD-ICA regarding BER, MSE and PAPR results. HICA method is showing the BER, and MSE performances significantly improved as compared state-of-art methods with the different antenna and FFT sizes using the QPSK modulation methods. However, the PAPR performance is not efficient using the HICA technique. HICA method failed to reduce the PAPR rate against the state-of-art methods. For future work, it will be interesting to try and check PAPR reduction methods with HICA to optimize the PAPR performance as well.

\section{REFERENCES}

[1] C. Shin, Jr., R. W. Heath, and E. J. Powers, "Blind channel estimation for MIMO-OFDM systems,” IEEE Trans. Veh. Technol., vol. 56, no. 2, pp. 670-685, Mar. 2007

[2] Yufei.jiang, xuzhu, enggeelim, yihuang. Orthogonal Sequences Based Multi-CFO Estimation and Semi-Blind ICA Based Equalization for Multiuser Comp Systems. Computer Science \&Information Systems. 2012; 9(4): 1385-1406.

[3] J Gao, X Zhu, AK Nandi. Independent Component Analysis for Multiple-Input Multiple-Output Wireless Communication Systems. Signal Processing. 2011; 91(4): 607-623.

[4] Chiu Shun Wong, Dragan Obradovic. Independent Component Analysis (ICA) for Blind Equalization of Frequency Selective Channels. 14thIEEE workshop Neural Networks for Signal Processing. 2003; 419-428.

[5] Obradovic D, Madhu N, Szabo A, Wong CS. Independent Component Analysis for Semi-Blind Signal Separation in MIMO Mobile Frequency Selective Communication Channels. IEEE International Joint Conference on Neural Networks (IJCNN). 2004; 1: 53-58.

[6] M Sifuzzaman, MR Islam, MZ Ali. Application of Wavelet Transform and its Advantages Compared to Fourier Transform. Journal of Physical Sciences. 2009; 13: 121-134.

[7] Karanpreet Kaur. Disceret Wavelet Transform based OFDM System using Convolutional Encoding. Master thesis. Patiala: Thapar university; 2014

[8] Xiangli Shao, Jian Chen and Yonghong Kuo, "Blind Channel Estimation for MIMO-OFDM Systems Based on Repetition Index", 2011 International Conference on Internet Computing and Information Ser-vices, IEEE, 2011

[9] Nima Sarmadi, Marius Pesavento, "Closed-Form Blind Channel Estimation In Orthogonally Coded Mimo-Ofdm Systems: A Simple Strategy To Resolve Non-Scalar Ambiguities", 2011 IEEE 12th International Workshop on Signal Processing Advances in Wireless Communications.

[10] Chao-Cheng Tu and Benoît Champagne, "Blind Recursive Sub-space-Based Identification of Time-Varying Wideband MIMO Channels", IEEE Transactions On Vehicular Technology, VOL. 61, NO. 2, FEBRUARY 2012

[11] Jian-Da Jiang, Tzu-Chiao Lin, and See-May Phoong, "New Sub-space-Based Blind Channel Estimation for Orthogonally Coded MIMO-OFDM Systems", 2014 IEEE International Conference on Acoustic, Speech and Signal Processing (ICASSP).

[12] Gamal Mabrouk Abdel-Hamid, Reham S. Saad, "Blind Channel Estimation Using Wavelet Denoising of Independent Component Analysis for LTE", Indonesian Journal of Electrical Engineering and Computer Science Vol. 1, No. 1, January 2016.

[13] Leila Sahraoui, Djmail Messadeg, Nouredinne Doghmane, "Analyses And Performance Of Techniques Papr Reduction For Stbc Mimo-Ofdm System In (4g) Wireless Communication", International Journal of Wireless \& Mobile Networks (IJWMN) Vol. 5, No. 5, October 2013

[14] P.Sunil Kumar, M.G.Sumithra, E.Praveen Kumar, "Performance Analysis of P APR reduction in STBC MIMOOFDM System", 2013 Fifth International Conference on Advanced Computing (ICoAC)

[15] Ying-Che Hung and Shang-Ho (Lawrence) Tsai, Senior Member, "PAPR Analysis and Mitigation Algorithms for Beam forming MIMO OFDM Systems", IEEE Transactions On Wireless Communications, Accepted For Publication, 2014

[16] S. Sujitha, R. Ramachandran, "Performance Analysis of PAPR Reduction in MIMO OFDM System Using Modified Constant Modulus Algorithm", IJAREEIE, Vol. 3, Special Issue 2, April 2014

[17] Sadhana Singha, Arvind Kumarb, "Performance Analysis of Adaptive Clipping Technique for Reduction of PAPR in Alamouti Coded MIMO-OFDM Systems", 6th International Conference on Advances in Computing \& Communications, ICACC 2016, 6-8 September 2016, Cochin, India

[18] Gourav Misra, Arun Agarwal, "A Technological Analysis and Survey on Peak-to-Average Power Reduction (PAPR) in MIMO-OFDM Wireless System", International Conference on Electrical, Electronics, and Optimization Techniques (ICEEOT) - 2016

[19] Feng Wan, Wei-Ping Zhu and M.N.S. Swamy, "Semi-Blind Channel Estimation of MIMO-OFDM Systems with Pulse Shaping”, 2008 IEEE International Symposium on Circuits and Systems

[20] LIU Haiyuan, SUN Jiancheng, "Blind MIMO-OFDM Channel Estimation Based on ICA and KRLS Algorithm, "2009 5th International Conference on Wireless Communications, Networking and Mobile Computing. 